\section{Remarks on the Hierarchical Organization of Fibers}

\section{Theo Odijk}

Faculty of Chemical Enginering and Materials Science, Delft University of Technology, P.O. Box 5045,

2600 GA Defft, The Netherlands

Recei ved December 18, 1995

Revised Manuscript Received March 28, 1996

Fibers are often organized in a hierarchical fashion. After quenching, the semifl exible macromolecules may coal esce into well-defined filaments, the filaments into microfibrils, the microfibrils into fibrils, and so forth (Figure 1). All or some of these slender, fibrillar objects may be physically and conceptually discerni ble entities; the fluctuation in the transverse dimension must then be small. A fibrillar hierarchy is often witnessed in natural materials ${ }^{1-2}$ like coll lagen ${ }^{3-6}$ and elastin ${ }^{7}$ fibers. Synthetic fibers spun from liquid-crystalline polymers have al so been characterized in terms of a hierarchical structure involving microfibrils. ${ }^{8-12}$ A central unresolved problem is how such a fibrillar organization comes about; why do the macromolecules not congeal into one more or less uniform amorphous mass without identifiable transverse length scal es? Fiber formation as a general problem of technological import, is sensitive to experimental conditions like the flow fiel ds imposed, the diffusi on of coagulative additives, inhomogeneities, and so on. This is much too complicated to analyze at present, so I wish to propose an idealized scheme based solely on lubrication forces which may explain why, for instance, mi crofibrils have enough time to coal esce into well-composed fibrils before the fibrils themsel ves start to adhere to each other. This occurs in spite of the rapid increase of Van der Waals forces with particle diameter. My arguments are very much qualitative; all numerical coefficients have been deleted even if they are of order 10. A full quantitative theory of fibrillar adhesion is a formidable task in view of orientation-translation coupling and the correlation of zipperlike longitudinal motion with transverse undulations. At a certain stage, the elasticity of the resulting fibrillar network also exerts itself as an energetic contribution holding back adhesion. The simplified lubrication route to fibrillar adhesion presented here, is obviously not free from limitations. Nonetheless, it is conceivable that experiments may be set up-the formation of fibers in concentrated suspensions of semiflexible polymers without additives in a quiescent state-which conform fairly closely to the present model.

First, it is of interest to consider the adhesion of filaments ${ }^{25}$ from a purely static point of view, as a problem in equilibrium statistical physics. In adhesion problems involving surfaces or lines, the fluctuations are generally large, so renormalization theory is the computational method of choice. ${ }^{13}$ Self-consistent arguments are sometimes valid provided the fluctuations are fairly small, as when filaments are confined within a potential well which is located at a secondary miniumum. ${ }^{14} \mathrm{~F}$ or the qualitative purpose of this Note, we simply balance ${ }^{15}$ the free energy of confinement against the unaveraged energy of attraction, here assumed to be a pure Van der Waals interaction.

The suspension we are dealing with is concentrated: the fiber volume fraction is typically about $50 \%$. The typical distance $h$ between two neighboring cylindrical surfaces is less than the (initial) filament diameter $D_{0}$.

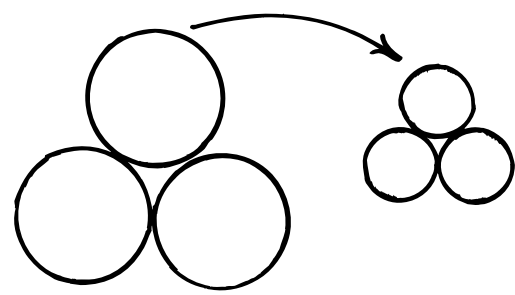

Figure 1. Example of fibrillar organization at two levels. Cross-sections of three well-defined microfibrils within a fibril; each microfibril in turn consists of three well-organized microfilaments.

The deflection length ${ }^{16} \lambda$ is then given by

$$
\lambda \simeq \mathrm{h}^{2 / 3} \mathrm{P}_{\mathrm{o}}^{1 / 3}
$$

where $P_{0}$ is the (initial) persistence length of a filament (see Table 1). The extensive free energy of confinement for a filament of length $L$ is ${ }^{16}$

$$
\mathrm{F} \simeq \mathrm{k}_{\mathrm{B}} \mathrm{TL} \lambda^{-1}
$$

with $\mathrm{k}_{\mathrm{B}}$ Boltzmann's constant and $\mathrm{T}$ the temperature. A decrease in the separation $h$ causes the entropy to diminish since configurational degrees of freedom are frozen out. But the Van der Waals attraction is steeper ${ }^{17}$

$$
\mathrm{U}_{\mathrm{attr}} \simeq-\frac{\mathrm{HLD}_{\mathrm{o}}^{1 / 2}}{\mathrm{~h}^{3 / 2}}
$$

where $\mathrm{H}$ is the Hamaker constant. Hence, on this view, the filaments simply adhere $(\mathrm{h} \rightarrow 0)$ and entropy does not present a barrier of much consequence. Equations 2 and 3 seem to provide a powerful case against hierarchical organization. In effect, we may suppose the adhesion among $\mathrm{n}$ filaments to be complete at a certain stage so that the newly formed filament behaves like a single unit of elastic material, without the possibility of sliding motion of any one of the original filaments. The bending elasticity and the persistence length $P$ are then not extensive properties (i.e. proportional to $n^{1 / 2}$ ) but rather scale as $\mathrm{P} \simeq\left(\mathrm{D} / \mathrm{D}_{0}\right)^{4} \mathrm{P}_{0} \simeq \mathrm{n}^{2} \mathrm{P}_{\mathrm{o}}$ in accordance with the usual Hookean law for bending rods ${ }^{18}(D=$ $n^{1 / 2} D_{0}$ new filament diameter). Therefore, fibrillar aggregation would entail a positive feedback loop. An increase in $n$ results in a stronger attraction (eq 3 ) but a weaker entropic repulsion (eq 2), which in turn results in a stronger tendency to enhance $n$, and so forth. In conclusion, equilibrium arguments would appear to favor an aggregational avalanche toward a state of complete adhesion without a tendency to form individual microfibrils or fibrils.

Equilibrium scenarios for the aggregation of rods have been formulated from other points of view. Percolative clusters have been analyzed by Warner, ${ }^{19}$ and Leung and Chandler, ${ }^{20}$ but these authors stressed the formation of open structures at fairly low concentrations, rather than the dense parallel arrangements seen in fibers. A micellar type of picture for parallel rodlike aggregates in dilute solution has been advanced by Van der Schoot ${ }^{21}$ who concluded that dimers might be stable sometimes, though, in general, the tendency is toward effectively infinite aggregation. On the whole, our reasoning above and that of others ${ }^{19-21}$ based on equilibrium states seem to preclude hierarchical organization. 
Table 1. Definition of Length Scales

$\mathrm{D}_{\mathrm{o}}=$ initial filament diameter

$\mathrm{P}_{\mathrm{o}}=$ initial filament persistence length

$\mathrm{D}=$ filament diameter at a certain level in the hierarchy

$\mathrm{P}=$ persistence length at a certain level in the hierarchy

$\lambda=$ deflection length at a certain level in the hierarchy

$\mathrm{h}=$ separation between two neighboring filaments

$\mathrm{L}=$ total contour length of a filament

I = length of a section within a cluster at a certain level in the hierarchy

Next, I necessarily turn to the dynamics of a concentrated suspension of highly oriented filaments attacted toward each other by Van der Waals forces, after the system is quenched by lowering the temperature or adding a poor solvent. Long-wavelength concentration fluctuations will be disregarded, as will be zipperlike adhesion and the elastic energy of the network, as the final state is reached. Aggregation will be assumed to occur by the approach of essentially parallel sections of filaments, which may be reasonable in concentrated solutions. Let us focus on what will be called a "cluster" consisting of two, three, or perhaps more filament sections approach each other. The longitudinal length I of some cluster is invariably much smaller than $L$, as any one filament, at some given moment, will belong to several consecutive clusters. The supposition will be made that I will typically be on the order of $\lambda$ : thelongwavelength cutoff is caused by thermal undulations. The filament sections within a cluster are aligned more or less parallel and orientation-translation coupling is simply disregarded.

The order of magnitude of the characteristic force driving a test section toward the central axis of the cluster it belongs to will be

$$
\mathrm{f} \simeq-\frac{\partial \mathrm{U}_{\mathrm{attr}}(\mathrm{I})}{\partial \mathrm{h}} \simeq \frac{\mathrm{HID}^{1 / 2}}{\mathrm{~h}^{5 / 2}}
$$

One or several filament sections of diameter $D$ pull in the test section. Within a cluster, thermal undulations are disregarded, which is feasible only if its longitudinal size I (h) decreases as the separation between its sections diminishes (see Table 1). However, our interest is not in the clusters themselves but only in that they remain long enough $(I \gg D)$ for the following qualitative picture to apply. Then, we have HID ${ }^{1 / 2} h^{-3 / 2}>>>k_{B} T$, so we neglect Brownian motion.

Let us first analyze the hydrodynamics of a cluster of only two sections (Figure 2). For small D, inertia may be neglected and the fluid of viscosity $\eta$ moves in accordance with the Stokes equation connecting the fluid velocity $\vec{v}$ with the pressure $p$

$$
\eta \Delta \overrightarrow{\mathrm{v}}=\vec{\nabla} \mathrm{p}
$$

Since the separations are generally small $(h \lesssim 1 / 2 \mathrm{D})$, a computation of the velocity field may be carried out in the lubrication approximation. ${ }^{22,23}$ A simplified scaling analysis involves a "surface of interaction". Equation 5 states that there are no special scales pertaining to the fluid between the two cylinders (Figure 2). Hence, the separation $\mathrm{h}$ is a relevant scale determining an inner region; the volume in which the fluid motion varies appreciably extends outward from height $\mathrm{h}$ to $\mathrm{a}$ height several times $h$. By geometry, the width of this volume is $h^{1 / 2} D^{1 / 2}$ and the surface of interaction has an area $A_{2} \simeq \mathrm{Ih}^{1 / 2} \mathrm{D}^{1 / 2}$ (see Figure 2 ; the same rule governs the Van der Waals attraction given by eq 3: the interaction between two half-spaces is multiplied by the

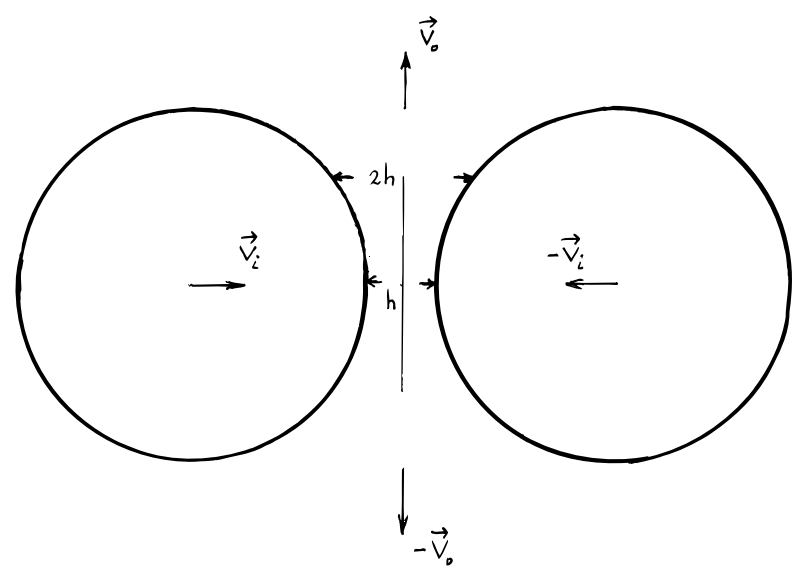

Figure 2. Two cylinders approach each other with velocity $\left|\vec{v}_{\mathrm{i}}\right|$ with respect to the fluid center axis. The "surface of interaction" is defined by the width between heights $h$ and $2 \mathrm{~h}$. The typical velocity of the fluid flowing out of the inner region is $\left|\vec{v}_{0}\right|$.

area of interaction). The pressure at the center axis of the fluid volume is $\left|f_{2}\right| / A_{2}$ which is exerted across a distance $h^{1 / 2} D^{1 / 2}$ so that eq 5 reduces to

$$
\left|\mathrm{f}_{2}\right| \simeq \mid \mathrm{D} \eta \mathrm{v}_{\mathrm{o}} \mathrm{h}^{-1}
$$

This determines the outward fluid motion $\vec{v}_{0}$ (Figure 2 ). The fluid is imcompressible so the velocity of approach $\vec{v}_{i}$ is given by $v_{i} h^{1 / 2} D^{1 / 2} \simeq v_{0} h$. Accordingly, the force needed to impose the velocity $v_{i}=-d h / d t$ is

$$
\left|\mathrm{f}_{2}\right| \simeq \mathrm{I}(\mathrm{D} / \mathrm{h})^{3 / 2} \eta \mathrm{v}_{\mathrm{i}}
$$

Equating this to eq 4, we finally get

$$
\mathrm{v}_{\mathrm{i}} \simeq \mathrm{H} / \eta \mathrm{hD}
$$

or

$$
\mathrm{h}=\left(\mathrm{h}_{\mathrm{o}}^{2}-\frac{\mathrm{Ht}}{\eta \mathrm{D}}\right)^{1 / 2}
$$

Therefore, if the starting position $\mathrm{h}_{\mathrm{o}}$ is a bit smaller than $\mathrm{D}_{0}$, on average, in a concentrated solution, the characteristic time needed for two sections within an initially defined cluster to adhere, is

$$
\tau_{2} \simeq \eta \mathrm{D}^{3} / \mathrm{H}
$$

A two-section encounter is probably rare, for three or more rodlike sections exert a greater inward force per unit length. ${ }^{24}$ A lubrication analysis of a three-cluster differs from that given above because a test rod now exerts a force on a body of fluid of area $A_{3} \simeq$ ID (Figure 3 ). The pressure is practically constant in most of the fluid, except in three inner regions across a distance of order $\mathrm{D}^{1 / 2} \mathrm{~h}^{1 / 2}$. Accordingly, eq 5 becomes

$$
\left|\mathrm{f}_{3}\right| \simeq \mathrm{l}(\mathrm{D} / \mathrm{h})^{3 / 2} \eta \mathrm{v}_{\mathrm{o}}
$$

Continuity dictates $v_{0} h \simeq v_{i} D$. The Van der Waals force (eq 4) yields a steady cylinder motion

$$
\mathrm{v}_{\mathrm{i}} \simeq \mathrm{H} / \eta \mathrm{D}^{2}
$$

and a characteristic time until adhesive contact given by 


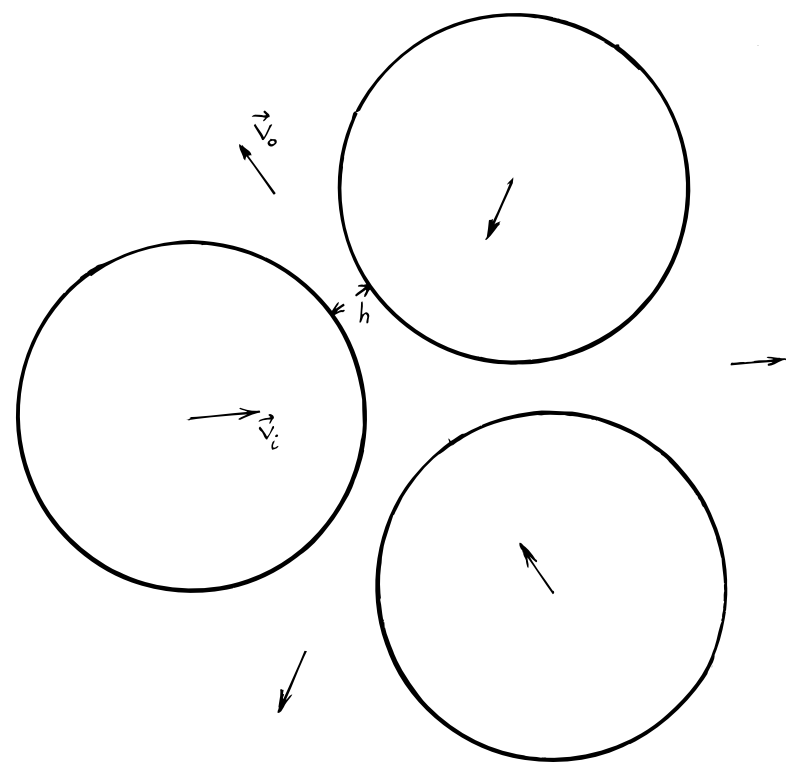

Figure 3. Mutual approach of three cylinders causing ejection of the fluid from the inner volume across three inner regions of lubrication.

$$
\tau_{3} \simeq \eta \mathrm{D}^{3} / \mathrm{H}
$$

An analysis of a four-cluster would give a similar result. Escape of the fluid "into the third dimension" at the filament ends has been neglected here. A comparison of the rates of transverse and longitudinal fluid transport shows that this is legitimate for long filaments ( ( $\mathrm{L} /$ $\left.D)^{2}(h / D)^{5 / 2} \gg 1\right)$. N ote, finally, that though, in reality, the aggregated cylinders are rough, the lubrication forces are perturbed by roughness only in the final stage of coalescence. The volume fraction is approximately constant during the entire process of aggregation, so at each stage $h \simeq 1 / 2 D$ at first. Hence, the qualitative estimate given by eq 13 should remain reasonable.

In the treatment here, fiber formation would be a problem in "punctuated lubrication". A typical cluster of, e.g., three rodlike sections would take a time $\tau_{3}$ or perhaps a bit longer to form a solid unit, i.e. a section of new filament, which together with two other similar filament sections would take about $2^{3} \tau_{3}$ to form a new unit, and so on. Thus, it would seem that there is enough time for rearrangement within the first cluster so as to form a new discernible filament section before the next stage of adhesion. Lubrication forces are strong enough to offset the strongly increasing tendency toward adhesive states under the influence of Van der Waals attraction. They slow down the process of fibrillar aggregation, possibly enough to rationalize, in part, the hierarchical organization found in fibers. In real life, there may well exist some effect not considered here, which singles out, e.g., one basic type of microfibril as a dominant mode in the aggregation process. The object of this Note has not been to give a complete view of fiber formation but rather to show that lubrication forces are definitely an impediment to a potential aggregational avalanche induced by static forces derivable from a free energy. Without such an impediment on all fibrillar levels, it is hard to see how well-defined fibers would ever form.

\section{References and Notes}

(1) Neville, A. C. Biology of Fibrous Composites; Cambridge University Press: Cambridge, U.K., 1993.

(2) Vincent, J. Structural Biomaterials; Princeton University Press: Princeton, N.J ., 1990.

(3) Katz, E. P.; Li., S. T. J . Mol. Biol. 1973, 80, 1.

(4) Baer, E.; Gathercole, L. J .; Keller, A. Colston Pap. 1975, 26, 189.

(5) Kastelic, J .; Galeski, A.; Baer, E. Connective Tissue Res. 1978, 6, 11 .

(6) Baselt, D. R.; Revel, J . P.; Baldeschwieler, J . D. Biophys. J . 1993, 65, 2644

(7) Urry, D. W. Ultrastructural Pathology 1983, 4, 227.

(8) Sawyer, L. C.; J affe, M. J . Mater. Sci. 1986, 21, 1897.

(9) Sawyer, L. C.; Chen, R. T.; J amieson, M. G.; Musselman, I. H.; Russell, P. E. J . Mater. Sci. Lett. 1992, 11, 69.

(10) Cohen, Y.; Thomas, E. L. Macromolecules 1988, 21, 433.

(11) Allen, S. R.; Farris, R. J .; Thomas, E. L. J . Mater. Sci. 1985, $20,4583$.

(12) J ackson, C. L.; Shaw, M. T. Polymer 1990, 31, 1070.

(13) Lipowsky, R. Phys. Scr. 1989, T29, 259.

(14) Odijk, T. ACS Symp. Ser. 1994, No. 548, 86.

(15) Helfrich, W.; Harbich, W. Chem. Scr. 1985, 25, 32.

(16) Odijk, T. Macromolecules 1983, 16, 1340.

(17) Mahanty, J .; Ninham, B. Dispersion Forces; Academic: London, 1976.

(18) Landau, L. D.; Lifshitz, E. M. Theory of Elasticity; Pergamon: Oxford, U.K., 1986.

(19) Warner, M. J . Chem. Soc., Faraday Trans. 1991, 87, 861.

(20) Leung, K.; Chandler, D. J . Stat. Phys. 1991, 63, 837.

(21) Van der Schoot, P. J . Phys. Chem. 1992, 96, 6083.

(22) Batchelor, G. K. Introduction to Fluid Dynamics; Cambridge University Press: Cambridge, U.K., 1967.

(23) Goddard, J . D.; Huang, Y. H.; Huang, L. C. In Proceedings of the 9th U.S. National Congress on Applied Mechanics; Pao, Y. H., Ed.; American Society of Mechanical Engineers: New York, 1982.

(24) Van der Schoot, P.; Odijk, T. J . Chem. Phys. 1992, 97, 515.

(25) The term "filament" will denote a fibrillar entity at whatever level within the hierarchy from now on.

MA951859H 